\title{
Identifiability Research of Generator Excitation System
}

\author{
Jian Zhang ${ }^{1, ~ a, ~ P e n g f e i ~ Z e n g ~}{ }^{1, b}$, Yali Zhang ${ }^{2, c}$ \\ ${ }^{1}$ Zhengzhou university, China \\ ${ }^{2}$ Luoyang Guanjie Push-Pull Cable Controller Co.,Ltd. Henan Province,China \\ azhangjian63@zzu.edu.cn, b184116842@qq.com, '867386964@qq.com
}

Keywords: Excitation system;Parameter identification;Study on identifiability.

Abstract. Found in the study of generator excitation system,by using the method of parameter identification for the model may well fit the system of input and output dynamic data, but some parameter identification result is very unstable or appear more solution, This phenomenon is known as the parameter identifiability. It has a close relationship between the parameter identifiability and trajectory sensitivity, and the parameters can not be identified simultaneously when the parameter sensitivity curves show the same phase or reverse phase changing.It is called associated parameters when the parameters can not be identified at the same time.For the associated parameters,then associated parameters were all estimated by assigning default value to some of the representative parameters.

\section{Introduction}

In the power system, the generator excitation system is mainly to fully play the role of a generator to increase the stability of the power system by excitation regulator.One of the key issues of power system digital simulation system is to establish accurate mathematical model and the generator excitation system is one of the important contents.

In the actual parameter identification, it is found that some parameters of the identification result is relatively stable and some parameters have great discreteness, but different combination of these parameters can well fit the measured results, that is, model parameters sometimes change,but model of dynamic image has little difference and test results is also very consistent. This phenomenon contains the problem of parameter identifiability [1].

Besides the error of modeling, there are two main factors which make the model parameters unrecogniziblity. One is that the parameter is not sensitive to the output, which is associated with the sensitivity of parameters (the concept of the sensitivity of parameters in the behind) and the another is that it is contain the relationship of implicit function among several parameters. Such as the impulse response function $\mathrm{g}(t)=p_{2} p_{3} e^{-p_{1} t}$,it can only identify the value of $p_{2} p_{3}$ rather than a separate value of $p_{2}$ and $p_{3}$, and it is because there is an implicit function between $p_{2}$ and $p_{3}$, that is, $p_{2}$ and $p_{3}$ have association.

According to the above, this paper put forward the way of recognizing the parameters of easy to identify and the parameters are associated according to the parameter sensitivity.For the associated parameters, firstly to elect associated parameters representative and assign default value.Then to use the rest associated parameters to fit the trajectory sensitivity of associated parameter representative.Finally to identify all of the parameters.

\section{Analysis method for the identifiability of excitation system}

Trajectory sensitivity refers to varying degrees of dynamic trajectory duo to small changes on parameter in system, and it reflect the relationship between the system trajectory and system parameter.Trajectory sensitivity is the derivative with respect to the parameter of trajectory[2].By using the perturbation method and using the median to calculate the derivative to obtain formulas of the trajectory sensitivity 


$$
J\left(\theta_{j}, k\right)=\frac{\theta_{j 0}}{2 y_{i 0} \Delta \theta_{j}}\left[y_{i}\left(\left(\theta_{1}, \theta_{2}, \mathrm{~L}, \theta_{j}+\Delta \theta_{j}, \mathrm{~L}, \theta_{m}\right), k\right)-y_{i}\left(\left(\theta_{1}, \theta_{2}, \mathrm{~L}, \theta_{j}-\Delta \theta_{j}, \mathrm{~L}, \theta_{m}\right), k\right)\right]
$$

Where $\mathrm{y}_{i}$ represents the track of the variate in the system $y_{i 0}$, represents the stable output of $\mathrm{y}_{i}, \mathrm{k}$ is the sampling time point, $m$ is the total number of parameters, $\theta_{\mathrm{j}}$ is the $\mathrm{j}$-th parameter, $\theta_{\mathrm{j} 0}$ represents the given value of $\theta_{j}$.

Suppose there are $\mathrm{n}$ parameters of the model connected by $\mathrm{m}$ effective implicit functions, that is

$$
\left\{\begin{array}{l}
\theta=\left\{\theta_{1}, \theta 2, \mathrm{~L}, \theta_{n}\right\} \\
\psi=\left\{\varphi_{1}(\theta), \varphi_{2}(\theta), \mathrm{L}, \varphi_{m}(\theta)\right\}
\end{array}\right.
$$

Where $m<n$.Assume any arbitrary $\varphi_{j}$ are derivable for any arbitrary associated parameter $\theta_{\mathrm{i}}$ and $\partial \varphi_{\mathrm{j}} / \partial \theta_{i}$ (non time variate) is a constant and recorded as $c_{i j}$, so

$$
\frac{\partial y}{\partial \theta_{i}}=\sum_{j=1}^{m} \frac{\partial y}{\partial \varphi_{j}} \frac{\partial \varphi_{j}}{\partial \theta_{i}}=\sum_{j=1}^{m} c_{i j} \frac{\partial y}{\partial \varphi_{j}}
$$

Obviously,the trajectory sensitivity of parameter can be expressed linearly by trajectory sensitivity of implicit function.

\section{Identification of associated parameters in excitation system}

Define $J(\theta(i))$ is a trajectory sensitivity matrix corresponding to parameter $\theta_{i}$ and $J\left(\theta_{\rho}\right)$ is linearly independent. Set $\theta_{\tau}(\kappa)$ is a represent in associated parameter set $\theta_{\tau}$ and $\psi(\kappa)$ is a parameter set corresponding to $\theta_{\tau}(\kappa)$ in unassociated parameter set $\theta_{\rho}$, which corresponds to the trajectory sensitivity matrix $J\left(\theta_{\tau}(\kappa)\right)$ and $J\left(\theta_{\mathrm{p}}\left(\psi_{\kappa}\right)\right)$ respectively,so $J\left(\theta_{\tau}(\kappa)\right)$ can be expressed by $J\left(\theta_{\mathrm{p}}\left(\psi_{\kappa}\right)\right)$ linearly[3].

The upper section shows that $J\left(\theta_{\tau}(\kappa)\right)$ can be expressed by $J\left(\theta_{\mathrm{p}}\left(\psi_{\kappa}\right)\right)$.If all the trajectory sensitivity of unassociated parameter $\theta_{\rho}$ join fitting, we can get

$$
J\left(\theta_{\tau}(\kappa)\right)=J\left(\theta_{\rho}\right) b_{k} .
$$

In theory, $J\left(\theta_{\tau}(\kappa)\right)$ are associated with $J\left(\theta_{\mathrm{p}}\left(\psi_{\kappa}\right)\right)$ and the fitting coefficient is not zero. $J\left(\theta_{\tau}(\kappa)\right)$ are unassociated with the rest of the parameters in $J\left(\theta_{\rho}\right)$ and the fitting coefficient is zero.But in the actual calculation, it can not be done that the fitting coefficient is zero when the parameters are unassociated.So it often set the threshold $\varepsilon$, and if $\left|b_{k}(\mathrm{i})\right|>\varepsilon, \theta_{\rho}(i) \in \psi(\kappa)$, or $\theta_{\rho}(i) \notin \psi(\kappa)[4]$.

According to the above method to calculate each subset in the associated set,such as $\psi(1), \psi(2), \mathrm{L}, \psi(\tau)$, and these parameter subsets constitute the associated parameter set $\psi$.

$$
\psi=\psi(1) \bigcup \psi(2) \bigcup L U \psi(\tau)
$$

\section{Assessment of identification results}

In practice,the assignment of the associated parameter representatives will always be deviations, which lead to the associated parameter identification results also exists errors. Therefore, it is necessary to evaluate the identification results of the associated parameters[5].

Assuming that $\theta_{\tau}(\kappa)$ deviates from the true value of the $\Delta \theta_{\tau}(\kappa)$, by the formula (4) can be obtained 


$$
\mathrm{J}\left(\theta_{\tau}(\kappa)\right) \Delta \theta_{\tau}(\kappa)=\mathrm{J}\left(\theta_{\rho}\right) \mathrm{b}_{k} \Delta \theta_{\tau}(\kappa)
$$

If $\Delta \theta_{\tau}(\kappa)$ is small, $\mathrm{J}\left(\theta_{\tau}(\kappa)\right) \Delta \theta_{\tau}(\kappa)$ can be used to approximate the variation of output trajectory when $\theta_{\tau}(\kappa)$ changes and $-b_{k} \Delta \theta_{\tau}(\kappa)$ can be used to offset the change of trajectory when $\theta_{\rho}$ changes.

Power system parameters always are non-linear,so when the assignment deviations of the associated parameter representatives are small,the assessment deviations and identification errors are close. It is easy to increase the number of weak associated parameters and the assessment deviations and identification errors will have relatively large differences when assignment deviations are large[6].So it is very important to select and assign the parameter representatives for identification result.

\section{Example analysis of excitation model}

Removing the limiting link, considering the type I excitation system for the linear model and it can be shown in figure 1.The model consists of 7 parameters, parameter names and values as shown in Table 1.

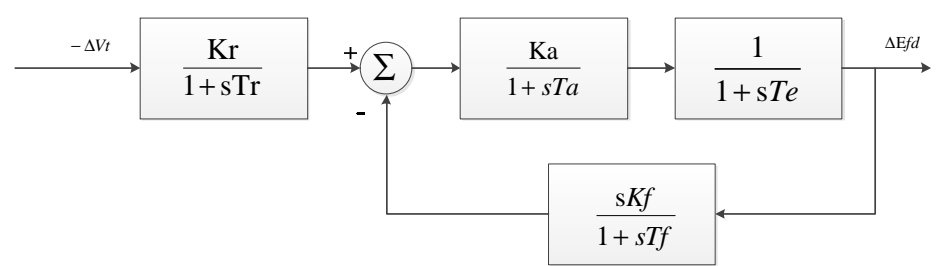

Fig.1 Transfer function block diagram for type I excitation model

Tab.1 Parameters for type I excitation model

\begin{tabular}{ccccccc}
\hline $\mathrm{K}_{\mathrm{r}}$ & $\mathrm{K}_{\mathrm{a}}$ & $\mathrm{K}_{\mathrm{f}}$ & $\mathrm{T}_{\mathrm{r}} / \mathrm{s}$ & $\mathrm{T}_{\mathrm{a}} / \mathrm{s}$ & $\mathrm{T}_{\mathrm{f}} / \mathrm{s}$ & $\mathrm{T}_{\mathrm{e}} / \mathrm{s}$ \\
\hline 1.00 & 20.00 & 0.04 & 0.04 & 0.04 & 0.70 & 0.80 \\
\hline
\end{tabular}

By using the transfer function method, it is found that the product of $\mathrm{K}_{\mathrm{r}} K_{a}$ and $\mathrm{K}_{\mathrm{f}} \mathrm{K}_{\mathrm{a}}$ can be identified but the $\mathrm{K}_{f} K_{r}$, and $\mathrm{K}_{\mathrm{a}}$ can not be identified individually. So these parameters are associated parameters. The shunt feedback is so weak that it can be ignored.So $\left(1+\mathrm{T}_{\mathrm{r}} s\right)\left(1+\mathrm{T}_{\mathrm{a}} s\right)=1+\left(T_{r}+T_{a}\right) s+T_{r} T_{a} s^{2} \approx 1+\left(T_{r}+T_{a}\right) s$. As a result, $T_{r}$ and $\mathrm{T}_{\mathrm{a}}$ work mainly through the $\mathrm{T}_{r}+T_{\mathrm{a}}$, they are associated parameters.

Calculating trajectory sensitivity of all parameters, selecting $\mathrm{K}_{f}$ and $\mathrm{T}_{\mathrm{a}}$ as representatives according to the method in the previous section and using the rest to fit trajectory sensitivity of these two parameters, the fitting coefficients are shown in the table 2.

Tab.2 Coefficients in trajectory sensitivity fitting

\begin{tabular}{cccrrr}
\hline parameters & $\mathrm{Kr}$ & $\mathrm{Ka}$ & $\mathrm{Tr}$ & $\mathrm{Tf}$ & $\mathrm{Te}$ \\
\hline $\mathrm{Kf}$ & -0.993 & 0.990 & $8.1 \times 10^{-4}$ & $-1.8 \times 10^{-4}$ & $-1.7 \times 10^{-4}$ \\
$\mathrm{Ta}$ & -0.063 & 0.061 & 1.123 & 0.006 & -0.073 \\
\hline
\end{tabular}

It is shown in table. 2 that coefficients $T_{r}, T_{f}$ and $T_{e}$ are close to 0 when fitting $\mathrm{K}_{f}$, while coefficients $K_{r}, \mathrm{~K}_{\mathrm{a}}, T_{f}$ and $T_{e}$ have larger errors when fitting $\mathrm{T}_{\mathrm{a}}$. This is because the former is strictly associated so that the fitting effect is better, while the latter is approximate associated when the higher-order terms are ignored.

Assigning different default values to $\mathrm{K}_{f}$ and $\mathrm{T}_{\mathrm{a}}$ to identify paremeters.Here setting three cases 
Case $1, \mathrm{~K}_{f}=0.04, \mathrm{~T}_{\mathrm{a}}=0.04$, that is, the default value takes from the true value.

Case $2, \mathrm{~K}_{f}=0.035, \mathrm{~T}_{\mathrm{a}}=0.035$, that is ,the default value takes a smaller deviation from the true value.

Case 3, $\mathrm{K}_{f}=0.025, \mathrm{~T}_{\mathrm{a}}=0.025$, that is , the default value takes larger deviation from the true value.

Tab.3 Identification results for model parameters

\begin{tabular}{rrlllllll}
\hline cases & $\mathrm{Kr}$ & $\mathrm{Ka}$ & $\mathrm{Tr}$ & $\mathrm{Tf}$ & $\mathrm{Te}$ & $\mathrm{K}_{\mathrm{r}} \mathrm{K}_{\mathrm{a}}$ & $\mathrm{K}_{\mathrm{a}} \mathrm{K}_{\mathrm{f}}$ & $\left(\mathrm{T}_{\mathrm{r}}+\mathrm{T}_{\mathrm{a}}\right)$ \\
\hline truevalue & 1.000 & 20.00 & 0.040 & 0.700 & 0.800 & 20.00 & 0.800 & 0.080 \\
case 1 & 1.006 & 20.09 & 0.036 & 0.712 & 0.814 & 20.20 & 0.804 & 0.079 \\
case 2 & 0.896 & 23.06 & 0.049 & 0.721 & 0.821 & 19.96 & 0.811 & 0.080 \\
case 3 & 0.607 & 32.65 & 0.068 & 0.724 & 0.816 & 19.96 & 0.816 & 0.084 \\
\hline
\end{tabular}

The above table shows that identification results are very close to real results when identifying the parameters $\mathrm{K}_{\mathrm{r}} K_{a}, \mathrm{~K}_{\mathrm{f}} \mathrm{K}_{\mathrm{a}}$ and $T_{r}+T_{a}$ as a whole. All other parameters identification results are very close to the real values when assigning true values to $\mathrm{K}_{f}$ and $\mathrm{T}_{\mathrm{a}}$. While the assignment values deviate from true values, the parameter identification results related with the associated paremeter representatives deviate from true values, but the parameters identification results unrelated with the associated paremeter representatives are close to true values.

\section{Conclusion}

Aiming at the problem of associated paremeters can not be identified individually in excitation system identification, analysing the linear correlation of trajectory sensitivity of associated paremeters and selecting and assigning default values to associated paremeter representatives according to calculating the fitting coefficients of trajectory sensitivity to identify all the paremeter.It shows that the method can improve the accuracy and robustness of the model parameter identification results.

\section{References}

[1]Wu Shuangxi,Zhang Boming,Bu Chuanwen. Identification and Assessment of Associated Parameters in Power Systems[J].beijing,china:Proceedings of the CSEE,31(22):73-79.

[2]Ju Ping .Nonlinear identification of power system[M].Nanjing,China:Hehai University Press, 1999:8-20(in Chinese).

[3]Cheng Xin.A Thesis Submitted in Partial of Fulfillment of the Requirements for the Degree of Master of Engineering[D].wuhan:Huazhong University Of Science And Technology.

[4]Hiskens I A,Pai M A.Trajectory sensitivity analysis of hybrid systems[J].IEEE Transactions on Circuits and Systems,2000,47(2):204-220.

[5]K. W. Wang, C. Y. Chung, C. T. Tse. Multimachine Eigenvalue Sensitivities of Power System Parameters [J]. IEEE Transactions on power systems, 2000, 15(2):741-747.

[6]Luo Yuchun. Study on Parameter Identification for the Generator's Excitation System[D].Nanjing:Nanjing University Of Science And Technology,2008. 